\title{
PROBLEMS ASSOCIATED WITH PATIENT CARE REPORTS AND TRANSFERRING DATA BETWEEN AMBULANCE AND HOSPITALS FROM THE PERSPECTIVE OF EMERGENCY MEDICAL TECHNICIANS
}

\author{
Smiljana Cuk, Georgia Southern University, sc07273@georgiasouthern.edu \\ Hayden Wimmer, Georgia Southern University, hwimmer@georgiasouthern.edu \\ Loreen M. Powell*, Bloomsburg University of Pennsylvania, lpowell@bloomu.edu
}

\begin{abstract}
While many hospitals have converted to electronic medical records, emergency medical services continue to employ paper-based reports. Furthermore, existing research focuses on challenges of information systems from the perspective of nurses, doctors, and hospitals. Little is known about the paper-based challenges facing emergency medical technicians. This study examined emergency medical technician's paper-based reports for potential problems that may occur if the transferred reports are in an electronic format. Additionally, this study conducted interviews of six emergency medical technicians about perceived benefits from electronic transfer of patient information for transferring patients. Results were positive as the emergency medical technicians liked to see a change from paper to electronic transfer of information. Emergency medical technicians also thought it was difficult to write the report while riding in the back of an ambulance, that information is lost during patient handover, and expressed a desire to follow-up on transferred patients.
\end{abstract}

Keywords: Healthcare Informatics, Electronic Medical Records, Medical Information Systems, Quick Reference Sheet, Patient Care Report, Data Transfer, Patient Handover, Emergency Medical Technicians

\section{INTRODUCTION}

Health informatics is a trending topic. Today, the exchange and storage of medical information is generally in electronic format. However, a big exception/problem in a system of electronic medical records is the patient care handover from the emergency medical technicians (EMTs) to the hospital personnel (Frellick \& Vega, 2016). Currently, most EMT patient care hand-off information is paper based (Starmer, et al., 2014). This research focused on the paper-based quick reference sheet (QRS) filled out by dispatched emergency medical technicians. The EMT QRS has multiple sections, which include: 1) medical care providers' information; 2) patient's personal information; 3) medication given to the patient; 4) vitals data; 5) other work completed during the call; and 6) a notes section. Currently, everything on this form is check boxes or lines for writing out the details. Figure 1 provides an image of an EMT QRS. When emergency medical services (EMS) arrive on a scene for a medical call, they have numerous tasks to accomplish including caring for the patient, calling the hospital and filling out a required QRS for the staff in the emergency department (ED). As a result, the handwritten QRS is difficult to read because it originated in the back of the ambulance while EMTs are rushing to the hospital. Moreover, the ED does not see the report with patient's information until EMTs arrive and hands-off the report. Additionally, a repeated EMT QRS may occur when the hospital nurse who received information via phone is not the same as the attending nurse in the ED, no hospital staff members available to receive the information and there may be a delay in transferring the patient's information. . . Based on the aforementioned issues, transfers of patients result in data loss, time delays, and miscommunication thereby decreasing patient care suffers (Duckw, 2016). If there are electronic QRS and patient care reports sent to the hospital then patients' outcomes may be enhanced.

There are numerous research studies on patient care hand offs form a nurse-to-nurse (Currie, 2002; Scovell, 2010), doctor's (Ye, taylor, Knott, Dent, \& MacBean, 2007), in-hospital staff perspectives (Owen, Hemmings, \& Brown, 2009) and general handover studies which suggest the use of technology for patient handoffs (Meisel, et al., 2015). However, little is known from an EMT's perspective regarding the procedures for transferring patients to the hospital (Yong, Dent, \& Weiland, 2008). Discovering more from the EMT perspective can aid in improving patient care. It is 
crucial to know first-responders' experiences and opinions. Their obligations and responsibilities with paperwork are an important aspect of the patient care process. A review of the literature indicates that there is limited to nonexistent research performed on a paper-based QRS and patient care reports from the EMT perspective.

This work has practical implications for the health informatics field, medical professionals and patients by uncovering factors that can help assist with improving the efficacy and transparency of patient information electronically. The remaining structure of this paper is the following: review of the literature, and methodology, findings, discussion and conclusion.

\section{LITERATURE REVIEW}

\section{Handover of Patients}

Sparsely investigated is a patients' handover process between the ambulance and emergency department staff. Jensen (2013) identified the major issues influencing the ambulance to ED handover and gave suggestions on how to optimize this process where they proposed solutions or optimization of the process. After completing the research, authors selected 18 as relevant out of 544. Most listed challenges regarding IT systems, as well as information loss during handovers. Transmitting electronic patient reports from the ambulance to a screen in the emergency room has been suggested. Researchers thought electronic data transmission would be a good idea because preparations prior to patient arrival could be improved, time spent on handover reduced, and information loss and typing errors would be cut down to minimum (Jensen, 2013).

There is limited information and understanding about efficiency of transferring information during patient handoffs from one health care provider to another. A collection of pre-hospital data sets are known to have an impact on the patients' outcomes hence it should be included in the EMS reports and recorded by emergency department staff. Carter (2009) conducted a research where all full trauma responses were video-recorded and watched to see whether the information was appropriately "transmitted" by the EMS providers. Next, $15 \%$ of randomly picked charts were checked for the same documented elements. Out of 1,536 possible data elements in 96 handovers, 473 elements transmitted and 329 were received. Mechanism of injury was the most commonly transmitted element, while prehospital hypotension was the least transferred element (Carter, 2009).

\section{Information Transfer}

Emergency departments either do not have a system to issue reports while patients are assessed in the hospital or the system that they have are not able to distribute a report due to delay in processing (Shelton, 2016). When it comes to distribution of emergency medical services reports, two different processes occur. The first process is produced by paramedics and transmitted to the emergency department by fax. The second process requires clerical staff to issue the fax report. In both cases, many factors cause delays in producing a patient care report.

At the moment of handoff from EMS to the emergency department, the information transfer may become highly degraded. Yet, the quality of care is greatly dependent on the transfer of patient information. Challenging environment errors occur frequently and have considerable impact on patient care. In order to be successful, EMS handoffs must rapidly and precisely convey information. However, verbal transfer is often only $50 \%$ accurate. The comparison of retained data and an external observer's objective assessment is, therefore, of the utmost importance. Panchal's research (2015) contained the following parameters: high acuity patients, patient arrival by EMS and external observers trained to evaluate the interaction. Trained observers recorded the communication between EMS and ED staff, their perception of the care transfer and evaluated the level of professionalism during the interaction. Collected data included heart rate, blood pressure, allergies and other points defined as crucial to patient care. The observers underwent a full training in order to be able to understand and pay attention to these key data points. If less than $75 \%$ of vitals or demographic data transferred, the handoff was a poor patient transfer. When it comes to EMS and ED providers' evaluation of quality, the results showed that absence of vital signs or demographics from the report was not as influential as the perceived professionalism. On the other hand, trained observers' evaluation was influenced 
by the presence or absence of vital signs or demographics (Panchal, 2015). Good handovers are associated with continuity of patient care, improvements in patient safety and decision-making (Wood, 2015).

As hospitals are implementing electronic health records, EMS are falling behind and are not keeping up with technology development. Less than a half of a country collects EMS data electronically but even then, that system is not integrated with hospital system. Electronic patient care reports (e-PCRs) still need to be printed off in the ambulance or hospital and hard copy is left with emergency department staff or it is later faxed to the hospital. Landman's research (2012) sought to characterize motivations for adoption of e-PCR systems, challenges associated with adoption and implementation, and emerging implementation strategies. Landman performed a qualitative study using semi-structured in-depth interviews with individuals representing multiple levels of the EMS agencies. Questions were targeting whether or not they had electronic report systems in place, its features, and barriers to adoption. Landman's result revealed that the primary reason for adoption of e-PCR systems was to support quality assurance. Maintaining the desired level of quality in a service with paper-based records is very challenging therefore is often not performed. By increasing availability of records with electronic patient care reports and automating them, quality assurance would be easier performed. Challenges with adoption of the e-PCR systems included: increased run times due to transitioning to a new system, lack of integration of e-PCR systems with existing hospital information systems, and deficiency of funding for state mandated e-PCR system implementations. Participants also shared the lessons they learned while transitioning and offered suggestions to agencies that still have not transitioned (Landman, 2012). This is the first study to consider the experiences of e-PCR system implementation and use from EMS perspective.

\section{Hospital Information Systems}

The Indiana Network for Patient Care (INPC) is a local health information infrastructure (LHII) that contains information from all of the major Indianapolis hospitals, clinics, and day surgery facilities. It collects around 2.7 million outpatient visits per year and carries 660 million separate results. The objective is to show the current state of the system and aid other communities with development. The Indiana Network for Patient Care system recognizes when patients check-in and allows physicians access to their files. When providers have staff privileges at more than one institution, they are granted access from those institutions as well. A second clinical service is delivering reports like laboratory, radiology, dictation, and other documents to office medical care providers whose names are tied to the report. This system also serves clinical, research, and public health functions. The Indiana Network for Patient Care has developed a community-wide clinical repository that provides cross-institutional access. Local health information infrastructure can be expected to improve efficiency and reduce cost growth, however, if its primary purpose becomes cost control, they will likely fail as did the community health information networks of the 1990s (McDonald, 2005).

While hospital information systems are widely being used, there is still no way of integrating pre-hospital patient care reports into that system. Majeed's study (2013) describes how a generic architecture could allow patient care systems to be integrated with a hospital's information system. Domain experts were questioned using semi-structured interviews to gather all of the requirements necessary for building software architecture. Next, for the creation of a software prototype, the architecture requirements were applied. To encode data, LOINC, ICD-10, and ICMP were used. Interviews produced requirements that led to creating a software architecture. All data was loaded into a common database using SQL scripts and individual items were mapped to semantic standards. Messages created from this structure were then sent to the hospital information system (Majeed, 2013).

By engaging Internet of Things (IoT) technologies in healthcare servicing, medical providers are able to see information resources online. Simply and quickly they can make emergency medical decisions and reduce the cost of the process. In Xu's research (2014), a semantic data model was projected for storing and interpreting data and then resource-based accessing method was designed for obtaining and processing data. Cloud computing served as a platform for synchronizing data across organizations. Results showed that the resource-based Internet of Things information accessing method is effective for supporting data accessing timely and ubiquitously (Xu, 2014). 


\section{Future Improvements}

Emergency medical services systems collect a large volume of data including patients' information (identification and medical history), type of incidents, symptoms and vital signs, and interventions. Although this could be a great source of information for conducting research, problem rises with reliability and extensiveness of data. Besides cases with incorrectly recorded information, bigger issues with data collection lies in unavailability of records concerning patient outcomes. An internal study accompanying published studies started by Weber (2015). An emergency medical services director that works in the emergency department matched each one of the selected group of patient care reports (containing usage of specific drug) with a hospital patient records pertaining same episode of care. After noting that in more than a half of all cases, patients were not diagnosed with a disease requiring that drug, usage of it was removed from ambulances. The results permitted emergency medical services agency to make a change to the treatment protocol. The ability to present concrete data convinced many who were initially against a proposed change (Weber, 2015).

\section{METHODOLOGY}

This research examined the current paper-based reports (QRS and subsequent patient care report) for the possibility of electronic transference format and its benefits. Figure 1 details a paper-based quick reference sheet (QRS). The QRS contains information about a patient such as demographics, oxygen, heart rate, blood pressure, medications, and most importantly, notes about the patient and condition.

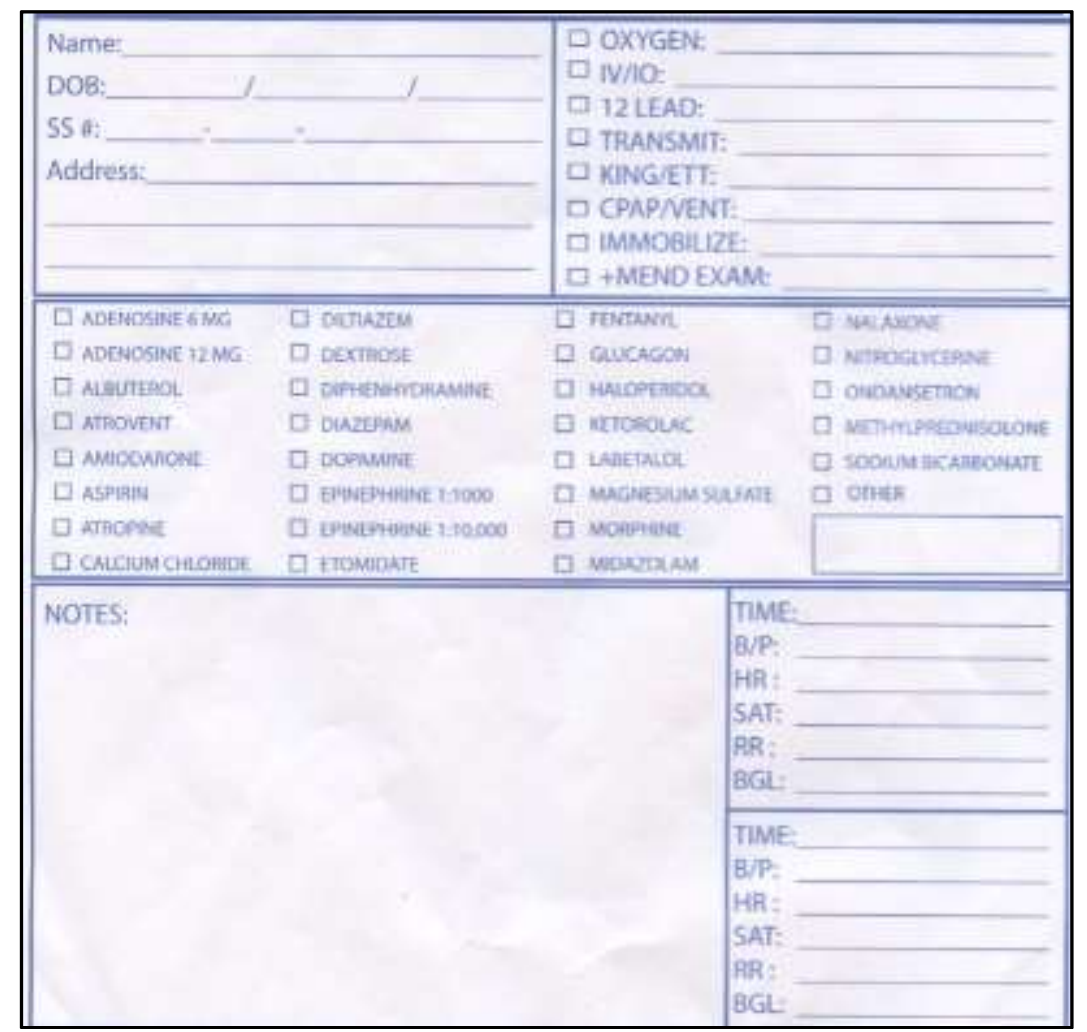

Figure 1. Paper-Based QRS

Additionally, this research examined the EMTs perspective of the paper-based QRS and subsequent patient care report into an electronic format. A qualitative method, which included an interview approach of EMTs was used. Many studies use qualitative methods to explore substantive areas about which little is known or about which much is known to gain better understandings (Stern, 1980). In our studies case, the only way to discover what occurs when an emergency call is placed is to talk to the people who handle the calls - EMTs. One of the most effective methods in 
qualitative research is open-ended questions because this style gives participants freedom to express their feelings and thoughts as well as provide researchers with rich and valuable information. With data extracted from the interviews, researchers can further learn and better understand the feelings and opinions of their subjects. In our case, we aim to understand issues surrounding the processes that out-of-hospital care providers, namely EMTs, face when dealing with antiquated paper-bases processes surrounded by the QRS and PCR.

\section{Interview Process}

This study conducted an open-ended question based interview with six EMTs throughout the Savannah, Georgia area. The interview questions started with one, main question - what problems are associated with the QRS and the PCR? A total of 18 open-ended questions were used (Please reference Figure B in the Appendix). Participants' responses were transcribed, coded and analyzed. To maintain confidentiality of responses, no individually identifiable records were kept. This research held face-to-face interviews at the local fire station's day room at convenient times for the participants. Each interview lasted approximately 30-40 minutes. Interviewees had an opportunity to express their thoughts and opinions on how their job effectiveness could escalate and patient's care be enhanced.

\section{FINDINGS}

This research interviewed six EMTs throughout the Savannah, Georgia area regarding the perceived benefits of electronic transfer of patient information for transferring patients. Additional EMT demographic data was not collected. Results showed several new problems with the QRS and patient care report that were not previously recognizable. Uncovering emergency medical technicians' perspectives were augmented with discovery of new issues. One of the issues with the QRS was that it is hard to write on a piece of paper in the back of an ambulance while rushing to the hospital. Several EMTs said, "I do not even bother completing it because most nurses do not look at it, or they return the report right back to us." In a few cases, the report got lost with other papers and medical components in an ambulance, which compromises patient's identity. One person mentioned that he would like to see more options on it so he can check it off instead of having to write it down.

When it comes to problems associated with transfer of patient care, other than the emergency room being too busy and hospital not having enough beds, there are verbal reports from EMS to the hospital staff. Once an ambulance arrives to the emergency department, a nurse is usually waiting to receive a transfer and some basic information about the patient. This is the second repeated paper report by EMTs because on the way to the hospital they have to call in and give the essential case information over the phone. Please reference except 1 regarding one interviewee opinion.

"When you are talking on the phone, other people are talking to them, you got other things going on in an ambulance, and there are a lot of confusion about trying to get this information across. If you could just send something like that, they will already be able to look at that patient records and see if they have ever been there and what is their history and medications, and know what you are coming in for today."

Excerpt 1. Issues Transmitting Information via Phone

Often, patients at the hospital are assigned several different nurses. As a result, another repeated verbal report or QRT. Sometimes, this can occur four or more times. This is time consuming and it is delaying the continuity of patients' care. One of the EMTs had a situation where no one passed the call-in information so nobody knew they were even coming. Please reference except 2 regarding one interviewee opinion.

"It makes us look unprofessional as well. One thing about both of our professions, patients have to be able to trust that we are going to give them the best care possible and if there is a breakdown in the continuity of care, then the patient and the patient's family are going to start losing trust in the care that we are trying to provide"

Excerpt 2. Issues Communicating Patient Information 


\section{Researcher Examination of paper Based Reports Results vs. Qualitative Results}

Upon examination of the EMT paper-based documents, the researchers note the following benefits may occur from transferring the documents to an electronic format. Specially, hospitals would already have patients' records and waiting time for checking patients in would be abridged. Nurses could be available sooner because they would not have to input patients' information into the database. EMTs could fill out only computer-based reports, which would leave them with more time for patients' care. Hence, by cutting down the waiting time in hospitals, patients' care would benefit, which is the most important. Additionally, an electronic system would be having the ability to check on transferred patients.

While the researchers found benefits in a transfer to electronic documents, it is important to note all EMTs unanimously agreed that switching from paper-based to electronic records would benefit, hospitals, EMTs and patients. Additionally, participants unanimously agree that their skills would improve if they had the opportunity to go back in the system to check on their patients. They would have a chance to see it they did something wrong, if they could have done something better, or maybe used a different drug. Universal opinion is that being able to see the outcomes would make them better practitioners, it would improve future patient care, and it would allow them to have closure and move on to the next call. For example, one of the participants stated, "Pain killer addiction is one of the biggest forms of drug abuse." That is another problem that could be solved with having one database $-83 \%$ of participants expressed their thoughts without even being asked an established question on this issue during interviews. "Drug seekers" are individuals that go from hospital to the hospital searching for pain medication. When they visit all of the hospitals in their surroundings, they can go back to the first one again because enough time has passed and they have been forgotten. One system would be able to keep a history of the prescriptions they have received and physicians would be able to "catch" these issues and stop the drug abuse.

Table 1 details the coding scheme employed in the analysis of issues with the paper-based QRS. The code, description, number of subjects that agree and number that disagree are displayed. From a qualitative analysis, we identified 27 codes for issues with patient care reports according to EMTs. Results indicate some issues are more important than other based on EMT agreement. The most prevalent issue is EMTs must repeat the written QRS verbally. Eliminating this issue would save time for the EMTs allowing them to focus on tasks directly related to patient care as opposed to duplicating efforts. Next, handing off the report to nursing staff presents challenges and frustration. One such example reveled through interviews is that many nurses discard the QRS or just give it back to the EMTs. Again, correcting this issue would improve efficiency of the patient hand-off process. Similarly, primary concerns for EMTs are drug abuse and drug seeking behaviors EMTs stated that while they can identify some drug seekers and communicate this information verbally, they are unable to identify drug seekers that are adept at concealing their intentions and that some drug seekers will travel over 50 miles in order to obtain prescription medications. In addition to the aforementioned issues, diverting EMTs to another facility causes wasted time and decreased patient care, as upon arriving at a medical facility, they are oftentimes required to redirect to another facility due to issues such as understaffing or an overflow situation in the emergency department. Should this information be communicated earlier in the process, patients could be more timely transported to the correct facility. The code ePCR2 is the only code where there was disagreement; however, this is actually a concern as the question relates to whether the EMTs ever send a full report to the hospital. Since five participants disagreed, this indicates that their full observations and efforts are never transmitted to the appropriate care facilities. Transferring the full report could aid in improved patient care. Analysis show that an average number of given verbal reports was 3.5. This indicates that EMTs must repeat their verbal report an average of 3.5 times per patient. This consumes EMTs' time and resources when the clinicians could review the patient care report during handoff. Table 1 and Table 2 present the remaining results. 
Table 1. Codes Identified from EMT Interviews

\begin{tabular}{|c|l|c|c|}
\hline \multicolumn{2}{|c|}{ Issues with reports } & Agree & Disagree \\
\hline Code & Description & 1 & 0 \\
\hline PCR1 & no time to write a report & 1 & 0 \\
\hline PCR2 & hard to write while riding in an ambulance & 1 & 0 \\
\hline PCR3 & hard to read the written report & 5 & 0 \\
\hline PCR4 & leaving QRS with a nurse & 2 & 0 \\
\hline PCR5 & QRS gets lost, patient's identity compromised & 1 & 0 \\
\hline PCR6 & more options needed & 2 & 0 \\
\hline ePCR1 & no time for computer based report & 0 & 5 \\
\hline ePCR2 & sending full report to the hospital & 1 & 0 \\
\hline CALL1 & calling hospital & 1 & 0 \\
\hline CALL2 & receptionist calling a nurse to answer a call & 1 & 0 \\
\hline CALL3 & hard to hear & 1 & 0 \\
\hline CALL4 & not paying full attention & 1 & 0 \\
\hline PASS1 & call information at the hospital not passed & 6 & 0 \\
\hline VRB1 & repeating report information verbally & 2 & 0 \\
\hline VRB3 & information lost during transfer & 2 & 0 \\
\hline TRN1 & diversion of patients, ED too busy & 2 & 0 \\
\hline TRN2 & waiting on a bed & 3 & 0 \\
\hline CARE1 & delaying continuity of patient care & 3 & 0 \\
\hline LOOK1 & unprofessional look & 1 & 0 \\
\hline LOOK2 & loosing trust & 2 & 0 \\
\hline FLUP1 & not being able to follow up & 2 & 0 \\
\hline FLUP2 & no personal improvement & 2 & 0 \\
\hline FLUP3 & not able to move on & 2 & 0 \\
\hline FLUP4 & treatment may hurt patient in a long run & 1 & 0 \\
\hline FQF1 & frequent fliers & 2 & 0 \\
\hline FQF2 & drug abuse/ seeking & 2 & 0 \\
\hline PCR & not filling it & 1 & 0 \\
\hline
\end{tabular}

Table 2. Average Number of Times the EMT Repeats a Verbal Report

\begin{tabular}{|c|l|c|}
\hline Code & Description & Average \\
\hline VRB2 & number of times verbal report is repeated & 3.5 \\
\hline
\end{tabular}

Table 3 details possible solutions to issues with the paper-based patient care report. First, sending the PCR electronically would facilitate the patient transition from EMT care to the medical facility. For example, EMTs stated if they could electronically transmit a summary or even cardiac data (heart rate and rhythm) the receiving facility could be more adequately prepared to accept and prepare for the patient. Often times, calling in this information results in miscommunication due to issues such as disconnected calls, noise interference from the ER or traffic, etc. Second, linking to the hospital would improve communications and efficiency. Ambulances that have electronic-based systems use them only internally because they are not integrated with hospitals' systems. Having a link or an integration would facilitate transferring patient data to the destination medical facility. Third, sending information between hospitals has the potential to improve patient care. For example, when transferring patients between multiple 
medical facilities, their information is left behind and has to be faxed over or brought in physically. Integrating the systems would provide clinicians the ability to get this data much faster and provide patient with continuous care.

Table 3. Possible Solutions Identified from Interviews

\begin{tabular}{|c|l|c|c|}
\hline \multicolumn{3}{|c|}{ Possible Solutions } \\
\hline Code & Description & Agree & Disagree \\
\hline SEND1 & sending PCR electronically & 6 & 0 \\
\hline LINK1 & linking up to the hospital & 6 & 0 \\
\hline LINK2 & being able to follow up on patients in a system & 6 & 0 \\
\hline LINK3 & sending information between hospitals & 6 & 0 \\
\hline FLUP4 & providing better care for future patients & 6 & 0 \\
\hline FLUP5 & personal improvement & 6 & 0 \\
\hline FLUP6 & having disclosure & 5 & 1 \\
\hline
\end{tabular}

In addition to electronic transmission solutions, there are also human related factors to consider. For example, EMTs interviewed unanimously want the ability to follow-up on a patient's condition. Excerpt 3 from one EMT details this desire. The majority ( 5 of 6 ) stated disclosure, or learning the outcome of a patient, would aid in being able to move on and completely focus on to the next call, and possibly improve job satisfaction. Also, EMTs unanimously agreed they would like the ability to check on transferred patients because that would improve future patient care as well as their skills and abilities to care for patients while under their supervision.

Many times, we are left wondering what happened. Unless we happen to be close friends with a nurse or the doctor, once we transfer care we lose track of the patient, we don't know what our interventions did in a long term until years later studies are done and they come back with stats. I had patients where I wondered for years what happened, did we save them, were they ever able to be discharged, did they ever resume a normal life.

Excerpt 3. Desire to Follow-up and Have Disclosure on Patient Outcomes

\section{DISCUSSION, LIMITATIONS AND CONCLUSIONS}

Multiple discussion topics could position future work. Discussions could open up on transitioning from paper-based report to electronically gathering and sending information. Problems that may arise from changing from one system to another are: costs of adoption, reluctance of some providers, training procedures, privacy concerns (Ndifon, Edwards, \& Halawi, 2016), as well as time delays during accommodation process, and need for an information technician position opening. Advantages will likely be cost reduction, improved quality of care through availability of patient information (Kilmon, Fagan, Pandey, \& Belt, 2008), performing quality assurance reports easier, faster data transfer, and of course, improved patients' outcomes. The social influence could play a bigger role on adoption of electronic medical records system than performance and effort expectancy (Wills, El-Gayar, \& Bennett, 2008). Three major forms of social influence are normative - pressure of a group on individuals to follow dominant group norms, informational - acceptance of other viewpoints during group meetings and events, and referent informational rejecting any other viewpoints (Lawson-Body, Willoughby, Hoffner, \& Logossah, 2014). Future research should also consider the cost of electronic access within rural areas with weak network infrastructures.

This research is not without limitations. First, since no demographic data were collected on the EMTs, it is difficult to assess the importance of training, experience and age with the outcome of the data. For example, perhaps a newly trained EMT who is technology savvy would have a different opinion versus one who has used the paper-based system for years and is not tech savvy. Additionally, the data sample was small in size and geographical location. Future research should also address these issues.

There is much room for improvement in the field of medicine, especially when it comes to transferring data. Integrating existing hospital information systems with ambulance systems should be a priority so that patient care 
reports and other important data could be easily and quickly sent between medical professionals. Information would then be accessible in seconds, rather than having time delays, data loss, and miscommunication. Sending QRS and PCR electronically to one integrated system would improve patients' outcomes. Solutions for implementing the QRS to the patient care report could be making it the first page of a full report. Once filled out, it can be sent to the hospital and later retrieved and continued without re-typing the same information.

\section{REFERENCES}

Carter, A. J. (2009). Information loss in emergency medical services handover of trauma patients. Prehospital Emergency Care, 280-285.

Chalasani, S. (2003). Web-based medical information systems. Issues in Information Systems, 59-65.

Currie, J. (2002). Improving the efficiency of patient handover. Emergency Nurse, 10(3), 24-27.

Duckw, R. L. (2016). Five Ways to Perfect the Ptient handoff. EMS World 45(11), 38-64.

Frellick, M., \& Vega, C. (2016). Can a Web-Based "Handoff" Tool Reduce Medical Errors? Medscape Education Clinical Briefs, Retreived from: http://www.medscape.org/viewarticle/868002.

Jensen, S. M. (2013). Handover of patients: a topical review of ambulance. Acta Anaesthesiologica Scandinavica, 964-970.

Kilmon, C. A., Fagan, M. H., Pandey, V., \& Belt, T. (2008). Using the task technology fit model as a diagnostic tool for electronic medical records systems evaluation. Issues in Information Systems, 196-204.

Landman, A. B. (2012, March 5). Prehospital Electronic Patient Care Report Systems: Early Experiences from Emergency Medical Services Agency Leaders. (K. M. Cresswell, Ed.) Plos One, 7(3), 1-8.

Lawson-Body, A., Willoughby, L., Hoffner, E., \& Logossah, K. (2014). Implementation factors influencing electronic medical records system. Issues in Information Systems, 323-333.

Majeed, R. W. (2013). Architecture of a Prehospital Emergency Patient Care Report System. MEDINFO, 1151.

McDonald, C. (2005, September). The Indiana Network For Patient Care: A Working Local Health Information Infrastructure. Health Affairs, 1213-1220. Retrieved from Health Affairs.

Meisel, Z. F. (2014). Optimizing the Patient Handoff Between Emergency. Annals of Emergency Medicine, 310317.

Meisel, Z., Shea, J., Peacock, N., Dickinson, E. P., Bhatia, R., Buharin, E., \& Cannuscio, C. (2015). Optimizing the patient handoff between emergency medical services and the emergency department. Annals of Emergency Medicine, 65(3), 310-317.

Ndifon, L., Edwards, J. E., \& Halawi, L. (2016). Impact of Electronic Health Records On Patient Outcomes. Issues in Information Systems, 187-196.

Owen, C., Hemmings, L., \& Brown, T. (2009). Lost in translation: Maximizing handover effectiveness between paramedics and receiving staff in the emergency department. Emergency Medicine Australasia, Retreived from http://onlinelibrary.wiley.com/doi/10.1111/j.1742-6723.2009.01168.x/full. 
Panchal, A. R. (2015). The Impact of Professionalism on Transfer of Care to the Emergency Department. The Journal of Emergency Medicine, 18-25.

Scovell, S. (2010). Role of Nurse-to-Nurse handover in patient care. Nursing Standard 24(20), 35-39.

Shelton, D. (2016). Availability of ambulance patient care reports in the emergency department. BMJ Quality Improvement Reports.

Starmer, A., Spector, N., Srivastava, R., West, D., Rosenbluth, G., Allen, A., . . et al. Group., I.-P. S. (2014). Changes in medical errors after implementation of a handoff program. New England Journal of Medicine., 1803-1812. Retreived May 13, 2017 from: https://www.ncbi.nlm.nih.gov/pubmed/25372088.

Stern, P. N. (1980). Grounded Theory Methodology: Its Uses and Processes. Journal of Nursing Scholarship, 20.

Strauss, A., \& Corbin, J. (1990). Basics of Qualitative Research.

Sundermann, M. L. (2015). Inaccuracy of patient care reports for identification of critical resuscitation events during out-of-hospital cardiac arrest. American Journal of Emergency Medicine, 95-99.

Weber, M. J. (2015). Why research is important. EMS World, 32-38.

Wills, M. J., El-Gayar, O. F., \& Bennett, D. (2008). Examining healthcare professionals' acceptance of electronic medical records using UTAUT. Issues in Information Systems, 396-401.

Wood, K. (2015). Clinical handovers between prehospital and hospital staff: literature review. Emergency Medicine Journal, 577-581.

Xu, B. (2014). Ubiquitous Data Accessing Method in Iot-Based Information System for Emergency Medical Services. IEEE Transactions on Industrial Informatics, 1578-1586.

Ye, K., taylor, D., Knott, J., Dent, A., \& MacBean, E. (2007). Handover in the emergency department: Deficiencies and adverse events. Emergency Medicine Australas, 19(433), 41.

Yong, G., Dent, A., \& Weiland, T. (2008). Handover fro peramedics: Observations and emergency department clinician perceptions. Emeregency Medicine Australas (20), 55-149. 


\section{APPENDIX}

1. What all do you need to do when taking a patient to the Emergency Department (ED) other than taking care of the patient?

2. When do you fill out the Patient Care Report (PCR)?

a. Where?

b. How difficult is it to do so?

3. What do you do with hand written PCR once the patient has been handed over to the ED?

4. Do you fill out any other reports? (yes/no)
a. (If yes) Which one?
b. When?
c. What is the purpose of that report?

5. I've noticed on the bottom of the PCR a message that the PCR is only a quick reference sheet and that full report will be sent to the ED upon completion. Is there a full report? (yes/no)
a. (If yes) Who is completing that report?
b. Is it sent? (yes/no)
c. (If yes) Who is sending it?

6. What information is verbally communicated during patient handover to the Emergency Department?
a. To how many people?
b. To whom?

7. Do you encounter problems when transferring care from EMS to ED? (please answer yes/no)

a. What problems do you most often encounter when transferring care from EMS to ED?

8. Are there problems associated with a paper-based Patient Care Report? (please answer yes/no)

a. What problems are associated with paper based Patient Care Report?

b. How could those problems potentially get solved or become less difficult?

9. Would it be easier or harder if all the information could be sent electronically to ED before getting to the hospital (easier/harder)?
a. On a scale from 1-10 with 1 being the easiest and 10 the hardest, how much easier/harder
handover would be if all the information could be sent electronically to ED before getting to the hospital?

b. How(Why) would it be easier/harder if all the information could be sent electronically to ED before getting to the hospital?

10. Would you like to be able to go back to the system and follow up on your patients' condition that you transferred from EMS setting to ED? (yes/no)
a. How would going back to the system to follow up on your patients improve patient care?
b. How would going back to the system to follow up on your patients improve your skills?
c. How would going back to the system to follow up on your patients improve your mental health? (ability to have closure)

11. How would having one database, connected to all hospitals, help you with sharing the PCR and other information?

12. Thank you for all that valuable information, is there anything else you'd like to add before we end?

Figure B. Interview Questions 